\title{
Nanosilicon for single-electron devices
}

\author{
H. Mizuta ${ }^{\text {a,b,* }}$, Y. Furuta ${ }^{\text {a,b,1 }}$, T. Kamiya ${ }^{\text {b,c }}$, Y.T. Tan ${ }^{\text {b,d,2 }}$, Z.A.K. Durrani ${ }^{\text {b,d }}$, \\ S. Amakawa ${ }^{\mathrm{d}}, \mathrm{K}$. Nakazato ${ }^{\mathrm{a}, \mathrm{b}}, \mathrm{H}$. Ahmed ${ }^{\mathrm{b}, \mathrm{d}}$ \\ ${ }^{a}$ Hitachi Cambridge Laboratory, Hitachi Europe Ltd., Cavendish Laboratory, Madingley Road, Cambridge CB3 OHE, UK \\ ${ }^{\mathrm{b}}$ CREST JST (Japan Science and Technology), Shibuya TK Bldg., 3-13-11 Shibuya, Tokyo 150-0002, Japan \\ ${ }^{c}$ Materials and Structures Laboratory, Tokyo Institute of Technology, 4259 Nagatsuta, Midori-ku, Yokohama 226-8503, Japan \\ ${ }^{\mathrm{d}}$ Microelectronics Research Centre, University of Cambridge, Madingley Road, Cambridge CB3 OHE, UK
}

\begin{abstract}
This paper presents a brief overview of the physics of nanosilicon materials for single-electron device applications. We study how a nanosilicon grain and a discrete grain boundary work as a charging island and a tunnel barrier by using a point-contact transistor, which features an extremely short and narrow channel. Single-electron charging phenomena are investigated by comparing asprepared devices and various oxidized devices. The optimization of grain and grain-boundary structural parameters is discussed for improving the Coulomb blockade characteristics and realizing room temperature device operation.
\end{abstract}

(C) 2003 Published by Elsevier B.V.

PACS: 81.07.Bc; 73.23.Hk; 85.35.Gv

Keywords: Nanocrystalline silicon; Single-electron device; Coulomb blockade

\section{Introduction—why nanosilicon?}

The progress of nanofabrication technologies over the last two decades as allowed us to explore a new research field of single electronics [1]. Single-electron charging or 'Coulomb blockade (CB)' phenomena are expected to appear in semiconductor nanostructures and have been adopted as a new principle for manipulating a small number of electrons. Both for memory and logic applications, single-electron devices (SEDs) have been studied intensively to overcome the inherent scalability issue of the conventional CMOS devices. A key building block for SEDs is the multiple tunnel junction (MTJ), which is composed of multiple tunnel barriers and

\footnotetext{
${ }^{*}$ Corresponding author. Present address: Department of Physical Electronics, Tokyo Institute of Technology, 2-12-1 Okayama, Meguroku, Tokyo 152-8552, Japan. Tel./fax: +81-3-5734-3591.

E-mail address: mizuta@pe.titech.ac.jp (H. Mizuta).

${ }^{1}$ Present address: Electronics, Information Systems and Energy Engineering, Osaka University, 2-1 Yamada-oka, Suita, Osaka 5650871, Japan.

${ }^{2}$ Present address: Institute of Microelectronics, 11 Science Park Road, Science Park 2, Singapore 117685, Singapore.
}

electron islands. As a general guideline, the MTJ should meet the following two requirements to show the $\mathrm{CB}$ effects at a temperature $T$ :

$R_{\mathrm{t}} \gg R_{\mathrm{Q}}=h / e^{2}=25.6 \mathrm{k} \Omega$,

$E_{\mathrm{C}}=e^{2} / 2 C_{\Sigma} \gg k_{\mathrm{B}} T$

to avoid quantum and thermal smearing of the electronic states confined in the electron islands. In Eqs. (1) and (2) $R_{\mathrm{Q}}$ is the quantum resistance, $R_{\mathrm{t}}$ is the zero-bias tunnel resistance of the (generally nonlinear) current-voltage characteristic of a tunnel junction, $E_{\mathrm{C}}$ is the charging energy, and $C_{\Sigma}$ is an island capacitance.

A number of fabrication methods of MTJs have been reported based on crystalline silicon. These structures may be classified into two groups in terms of the manner of defining electron islands: patterned electron islands and naturally formed ones. A patterned electron island with a lateral size of less than $10 \mathrm{~nm}$ has been realized by using a pattern-dependent oxidation (PADOX) technique [2]. This technique utilizes an increase in a band gap due to quantum confinement in an ultranarrow channel and a decrease in a band gap due to compressive stress in the channel after oxidation process 
[3]. Naturally formed structures exploit various kinds of local disorder in patterned nanoscale structures. In a heavily doped Si wire [4] a few tens of nanometers in width, randomly distributed dopant atoms cause potential fluctuations, and a linear chain of electron islands can form naturally [5]. Because of its simplicity this MTJ structure has often been used for making CB memory and logic devices. However, the $\mathrm{CB}$ oscillation can usually be observed only at temperatures below $77 \mathrm{~K}$ as the tunnel barriers are not high enough to confine electrons at high temperatures. A surface roughness induced MTJ has also been reported for a very narrow MOS channel [6], and the CB oscillation was observed at room temperature for this structure. Among these methods, PADOX is the most favorable as the size and position of an electron island can be controlled. However, key parameters of tunnel barriers such as the barrier height and thickness are not controllable with this method.

Compared with these methods, nanosilicon is an attractive bottom-up approach for forming ultra-small charging islands, and several fabrication techniques have been developed. The first method is to use a very thin poly/nanocrystalline (nc) silicon film with the size of the grains down to a few nanometer. The poly/nc-Si films can be formed either from an amorphous Si film with solid phase crystallization (SPC) or by using a very high frequency (VHF) plasma-enhanced chemical vapour deposition (PECVD) at a low temperature [7]. In the SPC films the individual grains are usually columnar shaped, and the grain boundaries (GBs) between adjacent grains contain carrier trap states due to dangling bonds. On the other hand, in the PECVD films, the individual grains are more spherical, and the GBs are formed by a-Si:H layers between grains. Another approach is to use porous Si [8] formed by using photoanodization of the $\mathrm{Si}$ substrate. The surface of the nc-Si islands formed in the substrate can be oxidised selectively by electrochemical oxidation. Formation of a linear chain of nc-Si islands with a diameter as small as $5 \mathrm{~nm}$ has been observed [9]. A more recent approach is an aerosol deposition technique, VHF plasma enhanced deposition of silane with a hydrogen gas pulse sequence [10]. This technique facilitates in separating the nucleation and crystal growth process and helps to fabricate nc-Si particles with diameter less than $10 \mathrm{~nm}$ and dispersions of $1 \mathrm{~nm}$ [11]. Particle diameter down to $3 \mathrm{~nm}$ has also been reported recently [12]. The interparticle tunnel barriers can be formed by in situ oxidation or nitridation in a controlled manner.

In this paper we focus on the first approach based on poly/nc-Si films. We investigate structural and electrical properties of nanosilicon grains and GBs and discuss optimisation of their parameters for room temperature SED operation.

\section{Single-electron charging phenomenon in nanosilicon}

The macroscopic properties of the poly-Si film with large Si grains have been studied extensively for the development of thin film transistors and static random access memories, and various process techniques have been established to increase the grain size and to improve carrier mobility. However, the microscopic properties of individual nanoscale grains and discrete GBs have not been studied in detail. In this work, we adopt a pointcontact transistor (PC-Tr) [13], which has a channel with both the length $L$ and width $W$ as small as the grain size $L_{\text {grain }}$ of the nano/poly-Si film. The electric characteristics for the PC-Trs must therefore reflect the properties of individual grains and GBs contained in the channel. Fig. 1 shows an SEM image of a PC-Tr fabricated in a 50-nm thick SPC poly-Si film. The inset (a) to Fig. 1 is a SEM image of a Secco-etched poly-Si film that shows the individual grains and GBs. From the SEM observation it was found that $L_{\text {grain }}$ in this particular film ranges from 20 to $150 \mathrm{~nm}$. PC-Trs with $W$ and $L$ from 20 to $50 \mathrm{~nm}$ were patterned by high-resolution e-beam lithography and electrically isolated by reactive ion etching. Electrical characterization was performed for both as-prepared PC-Trs and those oxidized at $1000{ }^{\circ} \mathrm{C}$ for $15 \mathrm{~min}$ in dry $\mathrm{O}_{2}$ ambient. An effective potential barrier height $q V_{\mathrm{B}}$ of GBs was extracted from a temperature dependence of the $I_{\mathrm{ds}}-V_{\mathrm{ds}}$ characteristics at between 200 and $300 \mathrm{~K}$ using the thermionic emission model.

The as-prepared PC-Trs [13] did not show any Coulomb oscillation at temperatures above $4.2 \mathrm{~K}$. From the TEM observation of grain structures, the tunnel capacitance $C_{\text {tunnel }}$ between two adjacent grains was estimated to be larger than $80 \mathrm{aF}$. In addition, the tunnel resistance $R_{\mathrm{t}}$ was evaluated to be similar to $R_{\mathrm{Q}}$. Therefore both $E_{\mathrm{C}}$ and $R_{\mathrm{t}}$ are not sufficiently large to observe the $\mathrm{CB}$ effect even at cryogenic temperatures. The barrier height $q V_{\mathrm{B}}$ extracted for the as-prepared PC-Trs, ranges from 30 to $80 \mathrm{meV}$.

In contrast, most of the oxidized PC-Trs show a clear Coulomb oscillation in the $I_{\mathrm{ds}}-V_{\mathrm{gs}}$ curves and a Coulomb

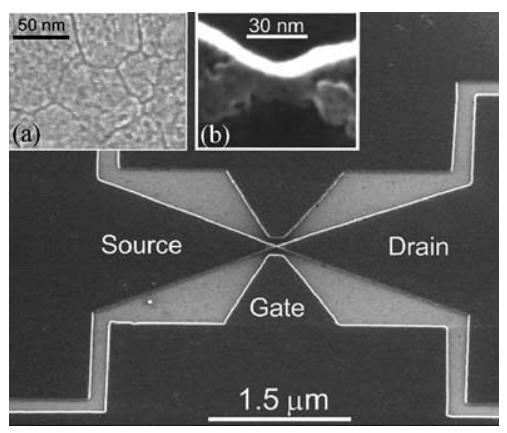

Fig. 1. SEM image of an as-prepared PC-Tr. The insets (a) and (b) show the grain structure of the poly-Si film and a blow-up of the channel region, respectively. 

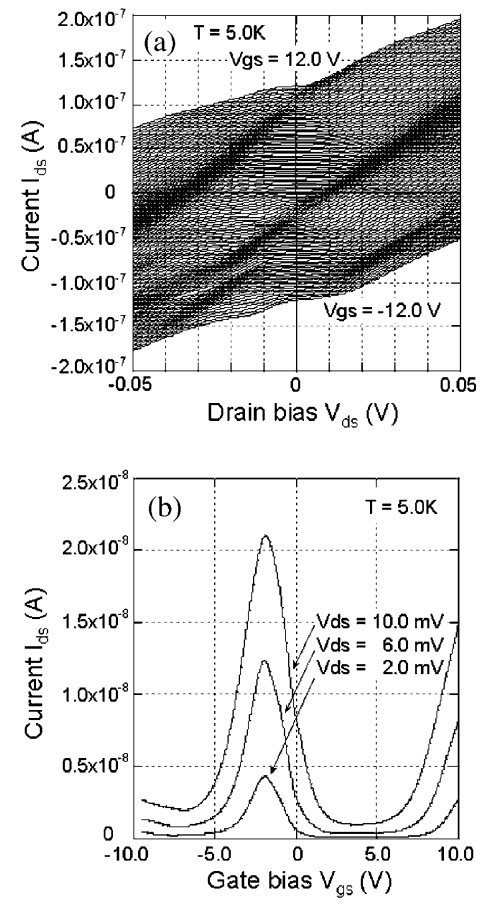

Fig. 2. (a) $I_{\mathrm{ds}}-V_{\mathrm{ds}}$ and (b) $I_{\mathrm{ds}}-V_{\mathrm{gs}}$ characteristics for an oxidised PC-Tr measured at $T=5 \mathrm{~K}$. Each $I_{\mathrm{ds}}-V_{\mathrm{ds}}$ curve in (a) is offset by $5 \mathrm{nA} / 100$ $\mathrm{mV}$ in $V_{\mathrm{gs}}$.

gap $V_{\mathrm{T}}$ in the $I_{\mathrm{ds}}-V_{\mathrm{ds}}$ characteristics associated with the $\mathrm{CB}$ effect [14]: Fig. 2(a) and (b) show the $I_{\mathrm{ds}}-V_{\mathrm{ds}}$ and $I_{\mathrm{ds}}-V_{\mathrm{gs}}$ characteristics for one of the oxidized PC-Trs. In the oxidized PC-Trs, the poly-Si film thickness decreased down to approximately $18 \mathrm{~nm}$ after the oxidation process [16]. This leads to a decrease in $C_{\text {tunnel }}$ and consequently increases $E_{\mathrm{C}}$ Although an increase in the mean $q V_{\mathrm{B}}$ was just $5 \mathrm{meV}$ for the oxidised PC-Trs, $R_{\mathrm{t}}$ was found to be by more than two orders of magnitude larger after the oxidation treatment because the GBs were oxidized effectively [17] and converted to suboxide. Both the increased $E_{\mathrm{C}}$ and $R_{\mathrm{t}}$ result in the appearance of the CB effect. The GB tunnel barrier thickness $d$ was estimated to be approximately $3 \mathrm{~nm}$ for the oxidized PC-Tr using a tunneling current calculation and observed value of $R_{\mathrm{t}}$. This is consistent with high-resolution TEM observation, which reveals that GBs were oxidized faster than crystalline grains and that some silicon suboxide layers were as thick as $2-3 \mathrm{~nm}$. The lateral dimensions of the grain that acts as the charging island were evaluated to be about $3 \mathrm{~nm} \times 10 \mathrm{~nm}$ by using a two-dimensional capacitance calculation and the observed $V_{\mathrm{T}}$ and Coulomb oscillation period $\Delta V_{\mathrm{gs}}$.

\section{Material optimisation towards room-temperature nano- silicon single-electron devices}

It was found from all the oxidized samples that both the $\mathrm{P} / \mathrm{V}$ ratio and $V_{\mathrm{T}}$ increase with $R_{\mathrm{t}}$ and that a good $\mathrm{P} / \mathrm{V}$ ratio is observed when the total zero-bias tunnel resistance of the device is over $1 \mathrm{M} \Omega$ [14]. If we assume $L_{\text {grain }} \cdot 3 \mathrm{~nm}$ and $d \cdot 1 \mathrm{~nm}$, a simple calculation [15] shows that $q V_{\mathrm{B}}$ should meet the following condition:

$q V_{\mathrm{B}} \cdot 260 \mathrm{meV}\left(\cdot 10 k_{\mathrm{B}} T\right)$.

Regarding the grain size, it is apparent that decreasing $L_{\text {grain }}$ further is needed to reduce $C_{\Sigma}$. For this purpose a nc-Si film prepared by a low-temperature VHF PECVD [7] was adopted instead of the SPC poly-Si film. A $20 \mathrm{~nm}$ thick nc-Si:H film was prepared from a $\mathrm{SiF}_{4}: \mathrm{H}_{2}: \mathrm{SiH}_{4}$ gas mixture at temperatures $\leqslant 300{ }^{\circ} \mathrm{C}$. From TEM observation it was found that the film contains grains with $L_{\text {grain }}$ from 4 to $8 \mathrm{~nm}$ uniformly distributed in a-Si:H matrix (Fig. 3(a)).

After defining the PC-Trs in the same manner, a multiple step oxidation process was applied, which was proposed to oxidize the a-Si:H selectively and to convert it to $\mathrm{SiO}_{x}(x<2)$ without increasing $L_{\text {grain }}[17,18]$. This is low-temperature oxidation $\left(650-750{ }^{\circ} \mathrm{C}\right)$ followed by high-temperature $\left(1000^{\circ} \mathrm{C}\right)$ annealing. A cross-sectional TEM showed that the grains remained the same in size despite the fact that the overall film thickness was reduced by $6 \mathrm{~nm}$ due to surface oxide formation. $q V_{\mathrm{B}}$ extracted for the multi-step oxidised nc-Si PC-Tr with $L=W=20 \mathrm{~nm}$ was as high as $173 \mathrm{meV}$ while that for the as-deposited nc-Si PC-Trs was only about $40 \mathrm{meV}$.

A clear CB oscillation was observed for the multi-step oxidised nc-Si PC-Trs: the $I_{\mathrm{ds}}-V_{\mathrm{gs}}$ characteristics observed for the device with $L=W=20 \mathrm{~nm}$ is shown in Fig. 3(b) [19]. The oscillation with an unchanged period persists up to room temperature although the P/V current ratio is gradually decreased as the temperature
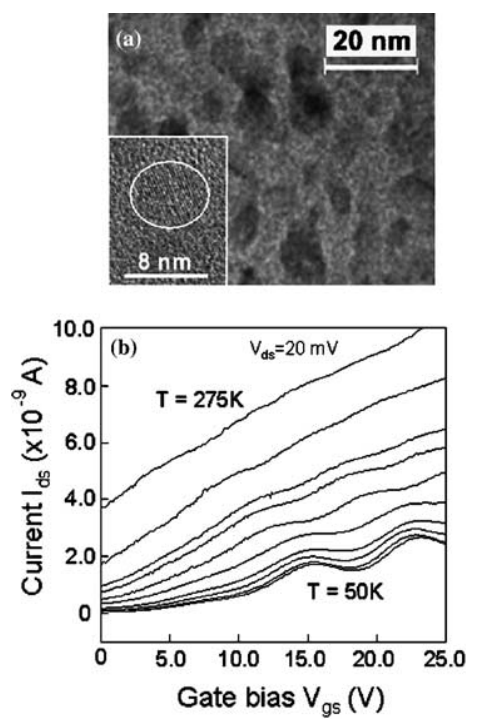

Fig. 3. (a) TEM image of the as-deposited PECVD nc-Si film with a blow-up of a nano-Si grain in the inset and (b) $I_{\mathrm{ds}}-V_{\mathrm{gs}}$ characteristics for the multi-step oxidised nc-Si PC-Tr. The temperature increases from 50 to $275 \mathrm{~K}$ in $25 \mathrm{~K}$ steps. 


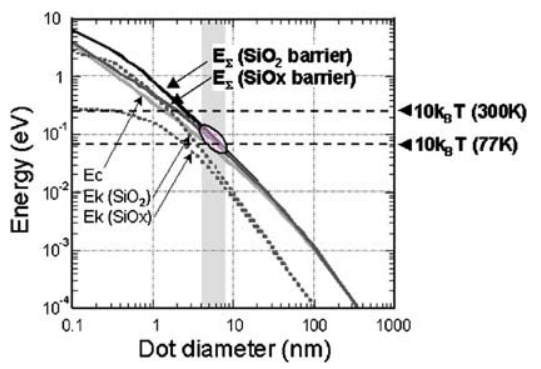

Fig. 4. Dot diameter dependence of the charging energy $E_{\mathrm{C}}$ the quantum confinement energy $E_{\mathrm{K}}$, and the total electron addition energy $E_{\Sigma}$ calculated for a $\mathrm{Si}$ sphere embedded in $\mathrm{SiO}_{2}$ or $\mathrm{SiO}_{x}$. An ellipse indicates $E_{\Sigma}$ corresponding to the nc-Si material used in the present experiment.

increases as shown in the inset to Fig. 4, and that for room temperature is still far too small for any kinds of device application. From a simple capacitance calculation combined with the observed $\Delta V_{\mathrm{gs}}$ and $V_{\mathrm{T}}, L_{\text {grain }}$ was estimated to be about $8 \mathrm{~nm}$ for this nc-Si PC-Tr, which is in good agreement with the TEM observation.

Even further reduction of $L_{\text {grain }}$ is needed for realising room temperature SED operation. To provide a more quantitative guideline, the charging energy $E_{\mathrm{C}}$ and quantum confinement energy $E_{\mathrm{K}}$ were calculated as a function of nc-Si dot diameter. $E_{\mathrm{C}}$ was calculated for a $\mathrm{Si}$ sphere embedded in oxide taking account of both self-capacitance and tunnel capacitance [20]. $E_{\mathrm{K}}$ was obtained numerically as the energy of the lowest quasibound state of an intrinsic Si quantum well with 2-nmthick oxide barriers: tunnel barrier heights of 3.0 and $0.26 \mathrm{eV}$ (Eq. (3)) were used for $\mathrm{SiO}_{2}$ and $\mathrm{SiO}_{x}$ barriers. Fig. 4 shows the dot diameter dependence of $E_{\mathrm{C}}$ (a light grey solid line), $E_{\mathrm{K}}$ calculated for both $\mathrm{SiO}_{2}$ and $\mathrm{SiO}_{x}$ (dotted lines) barriers and the corresponding total electron addition energies $E_{\Sigma}$ (black and dark grey solid lines). Because of the finite tunnel barrier heights, $E_{\mathrm{K}}$ approaches its maximum with decreasing dot diameter. A region of a diameter ranging from 4 to $8 \mathrm{~nm}$, which corresponds to the nc-Si film used above, is indicated as a shaded region. Two horizontal broken lines show the energy of $10 k_{\mathrm{B}} T$ with $T=300 \mathrm{~K}$ and $T=77 \mathrm{~K}$. These results indicate that

$L_{\text {grain }} \cdot 2 \mathrm{~nm}$

is needed to meet $E_{\Sigma} \cdot 10 k_{\mathrm{B}} T$ at room temperature, and to secure SED operation at room temperature, a further development is still needed towards 1-nm-scale size control of nanosilicon dots. However, in this region, the single-electron charging phenomenon in the nanosilicon material can certainly be a way of modulating current substantially at room temperature, and it is then important to develop new device structures that can operate based on the single-electron charging phenomenon combined with other mechanisms of controlling flow of electrons, such as the external electrostatic potential, tailored energy potential barriers [21], and energy quantization.

\section{Summary}

Nanosilicon has been investigated as a promising material for SEDs. We have shown that control of microscopic properties of GBs as a tunnel barrier is essential to improve $\mathrm{CB}$ characteristics by comparing asprepared and oxidised PC-Trs fabricated using the SPC poly-Si film and the PECVD nc-Si film. We have found that $q V_{\mathrm{B}}$ should be as large as $10 k_{\mathrm{B}} T$, and $L_{\text {grain }}$ should decrease down to less than $2 \mathrm{~nm}$ for any practical device applications at room temperature.

\section{References}

[1] H. Grabert, M.H. Devoret (Eds.), Single Charge TunnelingCoulomb Blockade Phenomena in Nanostructures, NATO ASI Series B, Plenum, New York, 1991.

[2] Y. Takahashi, H. Namatsu, K. Iwadate, M. Nagase, K. Murase, IEEE Trans. Electron. Dev. 43 (1996) 1213.

[3] S. Horiguchi, M. Nagase, K. Shirakashi, H. Kageshima, Y. Takahashi, K. Murase, Jpn. J. Appl. Phys. 40 (2001) L29.

[4] R. Smith, H. Ahmed, J. Appl. Phys. 81 (1997) 2699.

[5] G. Evans, H. Mizuta, H. Ahmed, Jpn. J. Appl. Phys. 40 (2001) 5837.

[6] H. Ishikuro, T. Fujii, T. Saraya, G. Hashiguchi, T. Hiramoto, T. Ikoma, Appl. Phys. Lett. 68 (1996) 3585.

[7] T. Kamiya, K. Nakahata, Y.T. Tan, Z.A.K. Durrani, I. Shimizu, J. Appl. Phys. 89 (2001) 6265.

[8] N. Koshida, H. Koyama, Appl. Phys. Lett. 60 (1992) 347.

[9] A.G. Cullis, L.T. Canham, P.D.J. Calcott, J. Appl. Phys. 82 (1997) 909.

[10] S. Oda, M. Otabe, Mater. Res. Soc. Proc. 358 (1995) 721.

[11] T. Ifuku, M. Otabe, A. Itoh, S. Oda, Jpn. J. Appl. Phys. 36 (1997) 4031.

[12] J. De Blauwe et al., IEDM Tech. Dig. (2000) 683.

[13] Y. Furuta, H. Mizuta, K. Nakazato, Y.T. Tan, T. Kamiya, Z.A.K. Durrani, H. Ahmed, K. Taniguchi, Jpn. J. Appl. Phys. 40 (2001) L615.

[14] Y. Furuta, H. Mizuta, K. Nakazato, T. Kamiya, Y.T. Tan, Z.A.K. Durrani, K. Taniguchi, Jpn. J. Appl. Phys. 41 (2002) 2675.

[15] T. Kamiya, Y. Furuta, Y.-T. Tan, Z.A.K. Durrani, H. Mizuta, H. Ahmed, in: Polycrystalline Semiconductors VII-Bulk Materials, Thin Films, and Devices, T. Sameshima, T. Fukui, H.P. Strunk, J.H. Werner (Eds.), Series 'Solid State Phenomena', Scitech Publ., Uettikon am See, Switzerland, 2003, pp. 351-354.

[16] Y.T. Tan, Z.A.K. Durrani, H. Ahmed, J. Appl. Phys. 89 (2001) 1262.

[17] T. Kamiya, Z.A.K. Durrani, H. Ahmed, Appl. Phys. Lett. 81 (2002) 2388.

[18] T. Kamiya, Y.-T. Tan, Z.A.K. Durrani, H. Ahmed, J. Non-Cryst. Solids 299-302 (2002) 405.

[19] Z.A.K. Durrani, T. Kamiya, Y.T. Tan, H. Ahmed, N. Lloyd, Microelectron. Eng. 63 (2002) 267.

[20] K.K. Likharev, Proc. IEEE 87 (1999) 606.

[21] K.K. Likharev, Appl. Phys. Lett. 73 (1998) 2137. 